doi.org/10.22498/pages.27.2.75

\title{
Holocene land cover and land use in South and Southeast Asia for climate modeling
}

\author{
Anupama Krishnamurthy', M.-J. Gaillard ${ }^{2}$, S. Prasad ${ }^{1}$ and K.D. Morrison ${ }^{3}$
}

Pondicherry, India, 11-14 September 2019

This four-day workshop of the working group LandCover6k (pastglobalchanges.org/landcover6k) hosted at the French Institute of Pondicherry emphasized hands-on training in pollen-vegetation modeling methods and fieldwork protocols for pollen-based landcover reconstructions (LC) and archaeologybased land-use mapping (LU). The training was provided by LandCover6k members to a group of mainly ECRs and also senior palynologists, ecologists, archaeologists, and historians working in South and Southeast Asia (45 participants).

The first day included presentations on:

- LandCover6k, its activities, and the state-of-the-art methods for achieving pollen-based LC reconstructions using the Regional Estimates of VEgetation Abundance from Large Sites (REVEALS) model (Sugita 2007).

- Archaeology-based LU mapping, including per-capita land-use estimates and other quantitative information, e.g. regarding domestic animals and crops (Morrison et al. 2018).

- Establishing standard chronologies for pollen records with an introduction to chronology using radiocarbon dating and Bayesian age-depth modeling (Blaauw and Christen 2013).

The group discussions and 20 poster presentations facilitated exchanges on regional initiatives for achieving the goals of LandCoverbk as well as an overview of a possible regional-scale data synthesis starting with homogenizing the attributions of pollen types and plant taxa of this region, including their ecological significance. Finally, Y. Subbarayalu gave a special lecture entitled "An independent LU data synthesis: Historical atlas of South India and its GIS database." distance-weighted vegetation data (Bunting et al. 2013; Fig 1) necessary to estimate Relative Pollen Productivities (RPPs, a key input in REVEALS) using the Extended R-Value (ERV) model, followed by discussions on the various regional challenges. A positive conclusion was the broad feasibility in tropical landscapes, given the new results from Southeast India (which will be published within the framework of Navya Reghu's PhD thesis) and Cameroon (Gaillard et al. 2019).

The remaining two days consisted of parallel sessions focused on LC and LU to achieve the overarching goals of the work packages WP-LC6 and WP-LU6 (pastglobalchanges. org/science/wg/landcover6k/scientific-goals), respectively.

The LC session included running ERV, REVEALS, and BACON age-depth models. The LU session focused on the application of the hierarchical scheme of global land-use classes used in LandCover6k and the effective use of Google Earth for LU mapping. The LU group capitalized on the participants' expertise by continuing work on correcting and completing paper maps produced during the regional LandCover6k initiative started a few months ago in Delhi under HoLa, an International Focus Group of INQUA HABCOM (inqua.org/commissions/ habcom/ifg). This allowed for the near completion of a basic land-use map for South Asia at $12 \mathrm{kyr} \mathrm{BP}, 6 \mathrm{kyr} B \mathrm{P}, 4 \mathrm{kyr} \mathrm{BP}$, and CE 1500 for publication. The plenary discussion sessions on the relevance of regional paleo and historical databases had a special focus on NEOTOMA (Williams et al. 2018), including a brief demonstration of the database. A special lecture by Shanti Pappu and Kumar Akhilesh provided perspectives on the changing archaeological landscape of South India through the Quaternary with public outreach insights.

During the final plenary, workshop participants discussed the available Holocene pollen data, including modern pollen-vegetation datasets from different ecological zones (WP-LC6) and the pressing need for a regional historical database (WP-LU6). It was decided to move forward with WP-LC6 in two ways in at least four distinct ecological zones where sufficient data and ongoing studies are available (Central Indian forests, Western Himalayas and Kashmir, Coastal Mangroves, Sri Lanka):

- Initiate fieldwork using the standard protocol for collection of pollen-vegetation data demonstrated during the workshop to obtain new estimates of RPPs for major plant taxa of the region.

- Use the numerous existing modern pollen samples to validate available RPPs from other regions, such as China and Europe ( $\mathrm{Li}$ et al. 2018; Mazier et al. 2012), for common plant taxa in e.g. the Western Himalayas and Kashmir, and test newly obtained RPPs from Southern India in the Central Indian forests.

It is feasible to extend these LC and LU goals in Cambodia, Vietnam, and Indonesia in the next year or two. In terms of databases, using available ones like NEOTOMA seemed most practical; however, the addition of a regional web portal would be useful. This workshop (pastglobalchanges.org/calendar/2019/127pages/1830) allowed the LU group (WP-LU6) to propose a roadmap for a regional historical database: to begin setting it up at the University of Pennsylvania where it is technically feasible to do so immediately and to eventually move it to a host institution in South/Southeast Asia.

\section{AFFILIATIONS}

Department of Ecology, French Institute of

Pondicherry, Pondicherry, India

'Department of Biology and Environmental Science,

Linnaeus University, Kalmar, Sweden

${ }^{3}$ Department of Anthropology, University of Pennsylvania, Philadelphia, USA

CONTACT

Anupama Krishnamurthy: anupama.k@ifpindia.org

\section{REFERENCES}

Blaauw M, Christen JA (2013) [Date accessed: 31.10.2019] Bacon Manual V2.2

Bunting MJ et al. (2013) Quat Sci Rev 82: 41-55 Gaillard M-J et al. (2019) 20th Congress of the

International Union for Quaternary Science. 25-31 July 2019, O-5083

Li F et al. (2018) Front Plant Sci 9: 1214

Mazier F et al. (2012) Rev Palaeobot Palyno 187: 38-49 Morrison KD et al. (2018) PAGES Mag 26: 8-9

Sugita S (2007) Holocene 17: 229-241

Williams JW et al. (2018) Quat Res 89: 156-177 